\title{
The Possibility of Diabetic Wound Healing Using Electro spun PLA Nano Fibers
}

\author{
Saeed Ahmadi Majd ${ }^{1 *}$, Mohammad Rabbani Khorasgani ${ }^{1}$, Mahna Mapar ${ }^{1}$, Mohammad \\ Asadollahi $^{1}$, Nahid Zarini Mehr ${ }^{1}$, Ardeshir Talebi ${ }^{2}$, Hamed Karimi ${ }^{3}$ \\ ${ }^{{ }_{1}}$ University of Isfahan, Isfahan, Iran \\ ${ }^{2}$ School of Medicine, Isfahan University of Medical Sciences, Isfahan, Iran \\ ${ }^{3}$ Islamic Azad University, Science and Research Branch, Tehran, Iran
}

Received: 17 November, 2017; Accepted: 27 November, 2017; Published: 29 November, 2017

*Corresponding author: Saeed Ahmadi Majd, University of Isfahan, Isfahan, Iran; E-mail: saeedmajd68@gmail.com

\begin{abstract}
Diabetes is a metabolic disorder demonstrated by hyperglycemia and is typically resulted from defects in insulin secretion and impaired pancreatic function. In general, wound infection is a common complication of diabetes and finding effective remedies (medications) and methods of diabetic wound healing has recently been of interests of researchers. In the present research study, PLA NANO fiber scaffolds are prepared by electro spinning in two different concentrations of $2 \%$ and $4 \%$. Indeed, electron microscopic scanning of this NANO fiber represents the achievement of NANO fibers with average diameter of $190 \mathrm{~nm} \pm 44 / 4 \mathrm{~nm}$ and $460 \mathrm{~nm} \pm 75 \mathrm{~nm}$ that encompass a uniform morphology. Given the fact that the PLA NANO fiber scaffolding with concentration of $2 \%$ has less average diameter smaller pores and higher biodegradability, hence, it has been selected to study wound healing. In this study, 18 male Wistar rats weighing $250-280 \mathrm{~g}$ are divided into three groups. First, two groups of rats become diabetic with streptozotocin (55mg / kg i.p.) and another group is monitored as a control type (healthy rats). After observing the symptoms of diabetes and hyperglycemia $(250-300 \mathrm{mg} / \mathrm{dl}$ in diabetic group), a wound is made on the back of neck of all four groups with diameter of $1 \mathrm{~cm}$ through surgical scissors. Then, wound is treated in the experimental group on a daily basis for 14 days by PLA NANO fiber scaffolding. Meanwhile, macroscopic and microscopic studies are adopted on $0,4,7,14$ and 7, 14 days respectively. The results revealed wound size reduction, epidermal gap decrement and reduction in wound treatment area in the treatment group compared to it in healthy groups. This, in turn, implies the positive impact of the NANO fibers in the process of wound healing.
\end{abstract}

Keywords: Diabetic; Wound healing; PLA; Nano fibers; Electro spinning

Abbreviations: PLA, poly lactic acid

\section{Background}

Generally, diabetic foot and diabetic foot ulcers are still considered as huge problems among diabetic patients. Diabetic foot problems are still largely unsolved despite many advances made in diagnosis and treatment of diabetes (1). Accordingly, wound healing depends on its blood supply as ulcers in diabetic patients are intensified due to vascular dysfunction and bacterial infection caused by diabetic ulcers that are not healed completely
(2). When a wound is created, a series of complex biochemical functions are quickly involved to rebuild damaged tissue but this restoration cannot be fully fulfilled because of the scar tissue (3). Therefore, adopting wound coverages to protect the wound, extract excess fluid from the wound surrounding, sterilize external microorganisms, and improve the appearance of accelerating healing process is required. In order to meet such requirements, coatings on wound healing must create a physical barrier while they are permeable against the passage of moisture and oxygen.

A number of methods have been used to cover wounds each having special features. In fact, special features should be considered to realize a proper wound covering remedy such as biocompatibility, biodegradability, accelerating recovery and healing process, preventing of secondary infection associated to ulcers or trauma, and preventing of wound symptom generation (scar)(4). Traditional and even common methods have shortcomings because they often lack at least one of the abovementioned features. The scaffold used wound covering should encircle biodegradability feature and degradation speed must be coordinated with generation speed of tissue. More importantly, these scaffolds should be highly porous to facilitate cell growth and penetration of nutrients (5). During the time period when the cell structure makes its own natural matrix, scaffold should provide a flawless structure and finally is released from new generated tissue as small molecules (6). Synthetic polymers used for acellular scaffold fabrication can be categorized as absorbable synthetic polymers, such as PCL, PLA, PEG, etc., and non absorbable synthetic polymers including polyurethane, nylon, polytetrafluoroethylene (PTFE), etc (7-9).

Poly-lactic acid is a biodegradable polymer with long substantial strings (10). Solid polymers are decomposed into available acids in the human body through delivering water to long polymer strands (11). The generated scaffolding in wound healing must be biodegradable, biocompatible and porous in addition to encompassing proper functioning feature. In this paper, we aimed to fabricate and study the characteristics 
mentioned in scaffolding PLA in order to apply it on skin wound healing in diabetic rats with streptozotocin (STZ) procedure.

\section{Methods}

\section{Identification of Poly Lactic Acid}

In general, gel permeation chromatography (GPC) is used to determine molecular weight and polymer dispersion index by the Central Laboratory of Isfahan University. The obtained results of GPC experiment revealed that the average molecular weight of poly lactic acid was about $300 \mathrm{KDa} 300$ with diffusion index of 1.575 .

\section{Structure of Polymer Scaffolds PLA using electro spinning method}

After determining PLA molecular weight through Gel permeation chromatography, polymer solutions are prepared to make electro spun NANO fibers. In order to prepare polymer solutions, PLA is dissolved in a solvent and is located in an ultrasonic bath for one hour (Figure.1a). For this purpose, electro spinning device is used that includes a syringe pump to inject the polymer solution and a source of high voltage between collector surface and feeder pump (12). Initially, PLA polymer is dissolved within solvents tri flora ethanol (TFE). Then, electro spinning solution is deployed on $5 \mathrm{ml}$ syringe including a needle with internal diameter of $(0.337 \pm 0.019)$. Moreover, clamp connected to power supply is joined to needle heading while rotating cylinder is connected to ground. In addition, solution injection rate is considered as $0.3-0.4 \mathrm{ml} / \mathrm{h}$ and operating distance is set as $9 \mathrm{~cm}$. Especially, NANO fibers formed on aluminum plate are collected which is located at a distance of $9 \mathrm{~cm}$ from the tip of the needle. Moreover, Electro spinning is executed in a lab environment with a temperature of $25^{\circ} \mathrm{C}$ for 6 hours (Figure.1b).

\section{NANO fiber scaffold morphology scanning within electron microscopy}

For providing electron microscopy, web NANO fiber sample with size of $(5 \times 10)$ is cut and deployed on the disc. The samples are then coated with gold material for 5 minutes and after that, NANO fibers morphology is examined by electron microscopy under $20 \mathrm{kv}$ voltage (Figure.1c).

\section{Determination of NANO fibers porosity}

Indirect calculation is adopted to determine porosity by applying polymer and NANO fiber scaffolds density. Furthermore, total porosity as percentage is obtained from porosity equation as follows.

Porosity percentage equation

$$
\begin{aligned}
\text { Porosity \% } & =\left\{1-d_{s} / d_{p}\right\} \times 100 \\
& =\left[1-\left\{m_{s} / v_{s} / d_{p}\right\}\right] \times 100 \\
& =\left[1-\left\{\mathrm{m}_{\mathrm{s}} / \pi \mathrm{d}^{2} \mathrm{t} / \mathrm{d}_{\mathrm{p}}\right\}\right] \times 100
\end{aligned}
$$

$\mathrm{m}_{\mathrm{s}}=$ scaffolds mass $) \mathrm{g}\left({ }^{\prime} \mathrm{v}_{\mathrm{s}}=\right.$ scaffolds volume $) \mathrm{cm}^{3}\left({ }^{\prime} \mathrm{d}=\right.$ =scaffolds diameter $(\mathrm{cm})$

$$
\mathrm{d}_{\mathrm{s}} \text { =scaffolds density ) } \mathrm{g} / \mathrm{cm}^{3} \text { ) 'dp=polymer density )g/ } \mathrm{cm}^{3} \text { ) }
$$

\section{NANO fibers diameter and pore size measurements by Image J software}

In this section, images obtained from electron microscopy image scanning are used to image analysis and measure the diameter of the NANO fibers and pores size. This measurement is derived via Image J software (Java Version). There is a scale bar within each image that must be calibrated through the Set scale option in initialization phase of image analysis. Moreover, NANO fibers diameter and pore size are analyzed manually and randomly. Before starting the process of image analysis, contrast and threshold of each image are optimized to identify top layer of fiber scaffolds. This is adopted to ensure that only top layer scaffold porosity will be measured. Information obtained from the measurement is then extracted and transferred to Excel for data analysis. Entirely, porous scaffolds are evaluated through this program and compared with theoretical data.

\section{Biodegradability test}

The concept of NANO fiber scaffold degradation refers to loss of scaffolds mass during chemical and biological reactions that occurs in the environment. In most cases, this deterioration is appeared due to hydrolysis of the main chain. For analysis of generated

NANO fiber scaffold degradation, samples are cut out in forms of circular pieces with a diameter of $1.88 \mathrm{~cm}$ and also weighed separately. Initially, NANO fiber scaffolds are rinsed with $70 \%$ ethanol and each side of scaffolds are sterilized for 20 minutes under the UV rays. Then third samples of each scaffold are maintained in cell cultivation container for 6 weeks within a solution of PBS 10 m-Molar with PH=7. Meanwhile, scaffolds are immersed in a solution of PBS. Moreover, cell cultivation containers are kept in a shaking incubator at $37^{\circ} \mathrm{C}$ temperature. Regularly, PBS solution is replaced with fresh solution every 2-3 days and subsequently 3 samples are removed from each scaffold after a week and then washed 3 times with deionized water and dried in a vacuum oven for 24 hours at ambient temperature weighing phase is executed.

\section{Animal model}

In this study, 18 outdoor male wistar rats weighing $280-250 \mathrm{~g}$ are purchased from medical science of Isfahan University and are kept in biology laboratory Group of Isfahan University under 12 hours illumination and 12 hours dark condition in temperature of $2 \pm 22$ degree in order to adapt them with new environment. These animals are then randomly assigned to experimental and controlled and negative control groups.

\section{Induction of diabetes in animal models}

In this section, streptozotocin (STZ) medicine manufactured by sigma firm is applied for induction of diabetes with dose of $55 \mathrm{mg} / \mathrm{kg}$ as injection to intraperitoneal. However, animal's blood 
glucose is measured three days after injection and animals with blood glucose greater than $250 \mathrm{mg} / \mathrm{dl}$ are considered as diabetic rats.

\section{Scarring in animal models}

In order to make scar, mice were first anesthetized by $50 \mathrm{mg} /$ $\mathrm{kg}$ of ketamine and $10 \mathrm{mg} / \mathrm{kg}$ of Xylazin. Moreover, their hairs on the back of the neck and between their shoulders are carefully shaved and wounds are created with dimensions of $1 * 1 \mathrm{~cm}^{2}$ (Figure.1d).

\section{Macroscopic studies}

According to this study, wounds are evaluated in terms of macroscopic model to determine the area of ulcer and wound healing trend for days of $0,4,7$ and 14 . It should however be noted that 7 and 14 days reflect significant importance in terms of wound healing process. Therefore, wound area in these days is determined with imaging and images analysis using the image processing toolbox of Photoshop software Cs6.

\section{Microscopic studies}

Specific samples of wound were released from each group on days of 7 and 14 for histological studies. Meanwhile, sample tissue to be stabilized is placed in container including $10 \%$ formalin and is transferred to pathology laboratory to prepare a tissue section slides. After complete stabilization of samples, tissue sections with a thickness of 5 microns are prepared and utilized Hematoxylin and eosin coloration with conventional methods. Finally, slide colored by a microscope (4Nikon, Japan) is investigated with zoom magnification of 10 and 40 (Figure.1e).

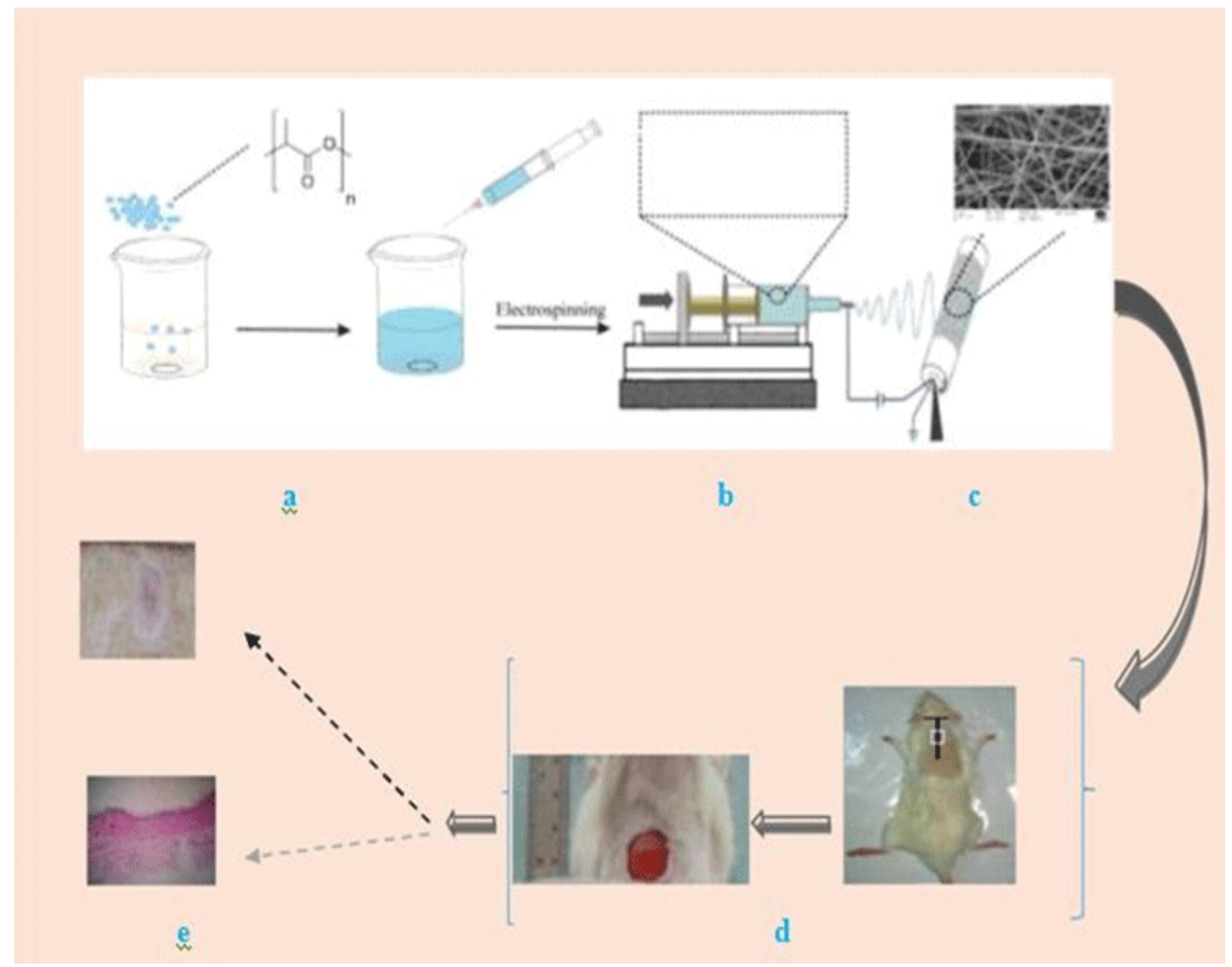

Figure 1: Schematic depicting, a: PLA solution, b: Electro spinning setup and phenomenon of electro spinning, $\mathbf{c}$ : electro spun fibers of PLA deposited on aluminum foil, d: Ulceration, e: Histological evaluation

\section{Biopsy of normal and diabetic rats pancreas tissue}

For the study and comparison of pancreas Langerhans islet beta cells in streptozotocin-induced diabetic rats, and normal rats, pancreatic biopsy of normal and diabetic rats were done and tissue samples were fixed in $10 \%$ formalin, then were stained by Hematoxylin \& Eosin and finally were evaluated by Leitz microscope with 400 times enlargement.

\section{Results}

\section{Electron microscopy}

Electron microscope and AFM images prepared from PLA scaffold surface is shown in (Figure.2). In this study, PLA polymer with concentrations of $2 \%$ is used fiber diameter at a concentration of $2 \%$ is determined $190 \mathrm{~nm} \pm 44 / 4$. 


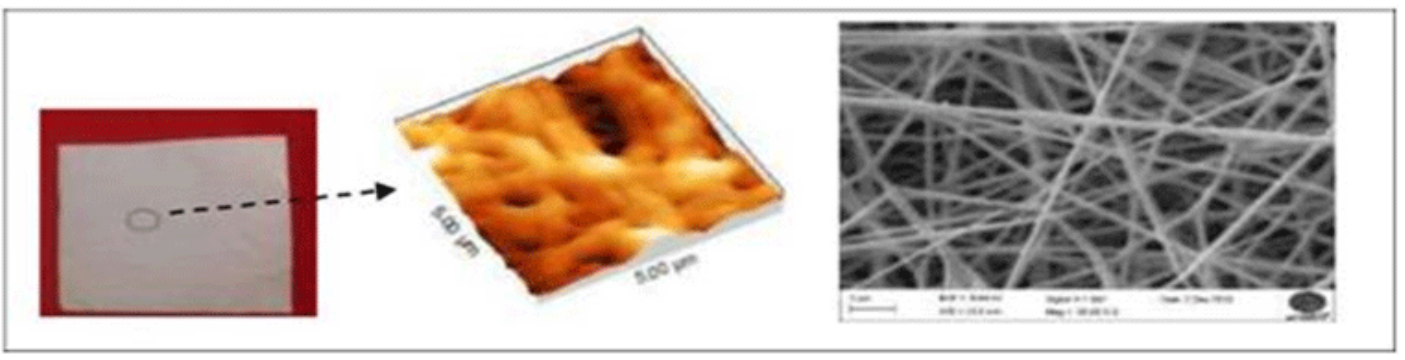

Figure 2: SEM image of poly lactic acid (PLA) Nano fibers prepared by electro spinning from with poly lactic acid concentrations of 2\%

\section{Porosity and pore size}

Porosity and pore size is reported in the table 1 . The theoretical porosity is reported based on average weight of diameter and thickness of the spun fibers and apparent porosity is obtained based on SEM images analysis.

Table 1: Porosity and pore size distribution of electro spun scaffolds

\begin{tabular}{|c|c|c|c|}
\hline & $\begin{array}{c}\text { Apparent } \\
\text { porosity\% }\end{array}$ & $\begin{array}{c}\text { Theoretical } \\
\text { Porosity \% }\end{array}$ & $\begin{array}{c}\text { The pore } \\
\text { size }\end{array}$ \\
\hline $\begin{array}{c}\text { Scaffolds of NANO } \\
\text { fibers\% }\end{array}$ & 89 & $99 / 17 \pm 0 / 15$ & $10 \mu \mathrm{m} \pm 4 \mu \mathrm{m}$ \\
\hline
\end{tabular}

\section{Biodegradability}

In this study, degradation speed is only dependent to morphology structure (size and diameter of the NANO fibers) due to utilization of PLA polymer. The PLA scaffold degradation speed with $2 \%$ concentrations is given in figures; as it is evident from figures, PLA scaffold degradation speed with $2 \%$ concentrations is higher which is induced as a result of lower diameter of NANO fibers produced through electro spinning process (Figure.3).

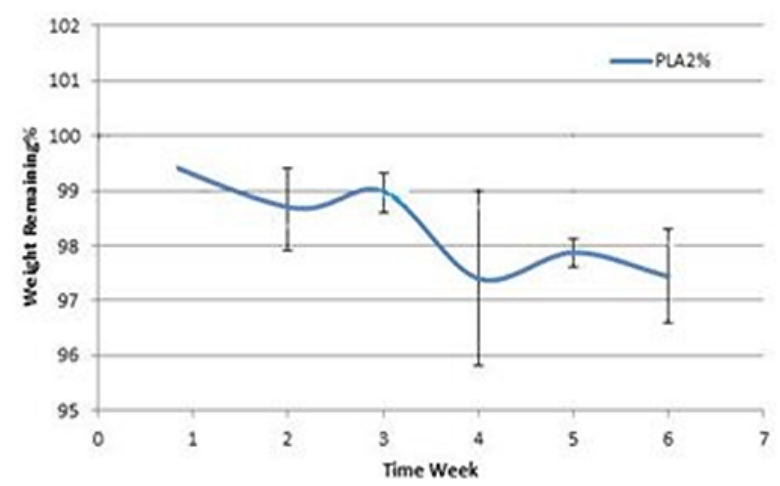

Figure 3: Weight remaining of PLA scaffolds after in vitro degradation for 6 weeks

\section{Induction of diabetes by streptozotocin}

Streptozotocin at dose $60 \mathrm{mg} / \mathrm{kg}$, resulted in high mortality in test group (11death per 25 rats), Therefore the dose: 55 $\mathrm{mg} / \mathrm{kg}$ was replaced it and diabetes developed within 3 days. The mortality rate significantly reduced (3death per 25 rats). Pancreatic biopsy of normal and diabetic rats confirmed that the islet and cells were destroyed due to the effect of Streptozotocin in diabetic rats. The comparison of these pictures shows that the tissue of pancreatic Langerhans and the beta cells of diabetic rats have been degenerated irreversibly (figure.4). The results of measuring of rats body weights indicated that average of body weight in diabetic rats reveals loss of weight and thinness in diabetic adult rats (figure.5). The blood glucose measurement before and after diabetes revealed that: the levels of glucose in healthy adult rats was $101 \pm 5 \mathrm{mg} / \mathrm{dl}$, But in diabetic rats was measured as $374 \pm 10 \mathrm{mg} / \mathrm{dl}$ (figure.6).

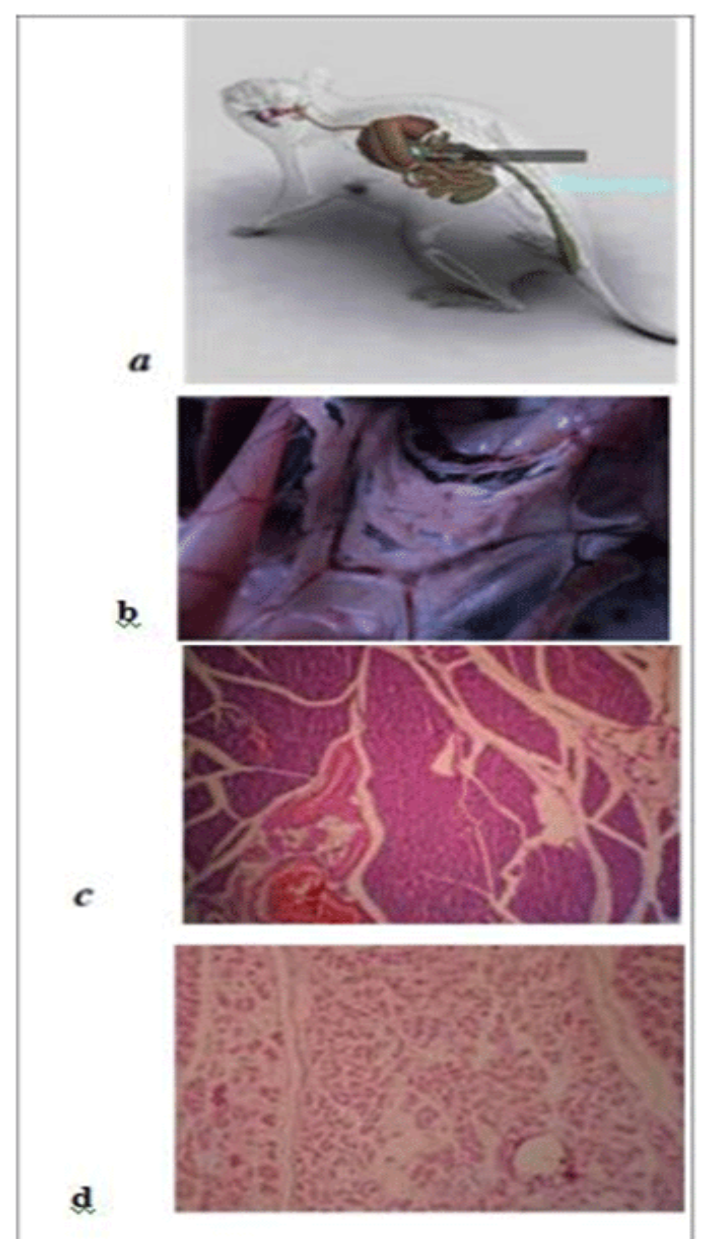

Figure 4: a: Anatomic relationships of the pancreas with surrounding organs and structures $\mathbf{b}$ : An isolated rat pancreas $\mathbf{c}$ : Pancreatic biopsy of normal rats d: Pancreatic biopsy of diabetic rats that confirms the necrosis of islets and cells due to the effect of streptozotocin 


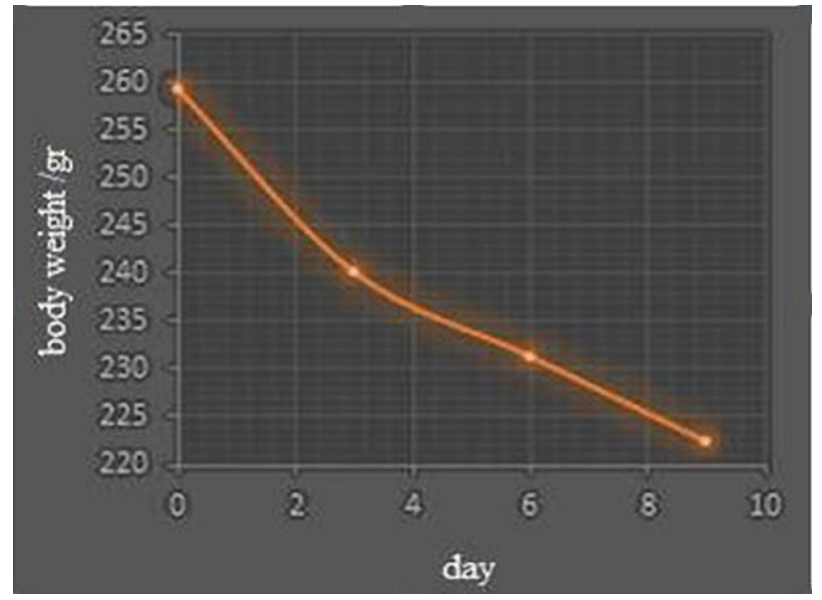

Figure 5: Shows continuous changes in average of body weight in diabetic rats in days $0,3,6$ and 9

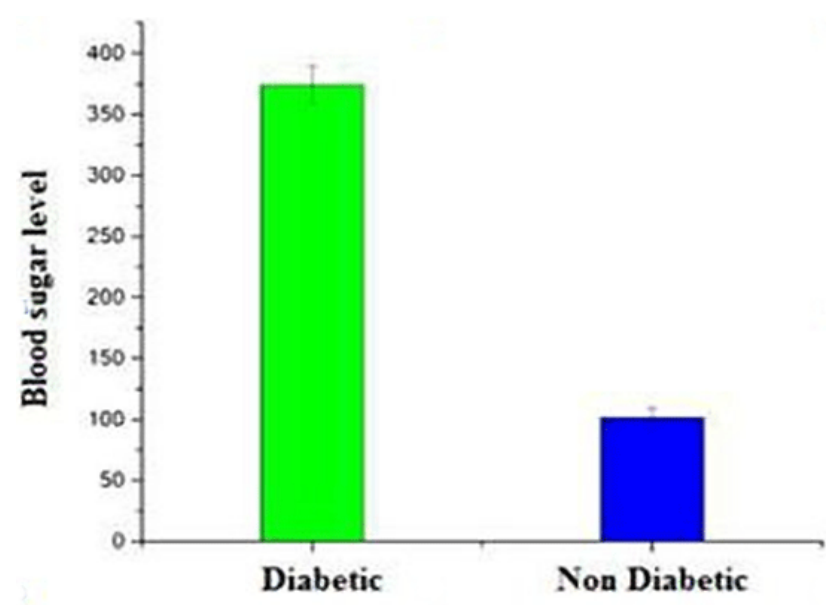

Figure 6: Shows the changes of average level of glucose in serum of diabetic and Non diabetic rats

\section{Macroscopic assessment of wound}

The average area of wound in diabetic group on days 7 and 14 is $93 \pm 4.25$ and $27.045 \mathrm{~mm}^{2}$ respectively. Moreover, average area of wound in treated group diabetic group with PLA NANO fibers on days 7 and 14 is $64 \pm 2.37$ and $27.045 \mathrm{~mm}^{2}$ respectively. According to comparison of average area of ulcers treated with diabetes control it can be concluded that there are significant differences between them $(P<0.05)$ (figure.7-8).

\section{Microscopic evaluation of wounds healing}

In the experiment group (PLA Nano fiber), histological findings indicate the reduction in the wound size and epidermis gap and dermis lesion surface length in the $7^{\text {th }}$ and $14^{\text {th }}$ days compared to the diabetic and healthy control groups (figure.7). This issue indicates its positive effect on the process of wound healing (figures.9-11).

\section{The microscopic pilot group in the seventh day}

There were no immediately apparent changes in the panniculus. On the seventh day, the wounds were open and featured with a pack of infiltrated inflammatory cells. The keratinocyte layers at the wound edges got thicker and the panniculus was not observed in the wound. In study of tissue sections marked with $\mathrm{H}$ \& E gap (the epidermal gap is not restored and the epithelial lining is developing and the epidermal gap length and dermal area in the wound is less than the control group. It has been seen that in granulation tissue, the cell congestion is more and the blood vessels are more repeated than those in the control group (figures.9-11).

\section{The microscopic pilot group in the fourteenth day}

Re-epithelialization was perfect in most of the wounds on the fourteenth day when high cellularity was still obvious and certain new collagens could be seen in the wounds. By day 14, the wound areas became shorter and dense granulation tissue was present. By day 32, cellularity significantly decreased and scar tissue was seen at the wound site. In the investigation of tissue, sections stained with $\mathrm{H} \& \mathrm{E}$ gap (the gap) epidermal layer has been restored and the remaining scar was not seen on the wound. The epidermal gap length in the wound has been reached 0 and epidermal area in the wound area is less than that in the control group (figures.9-11).

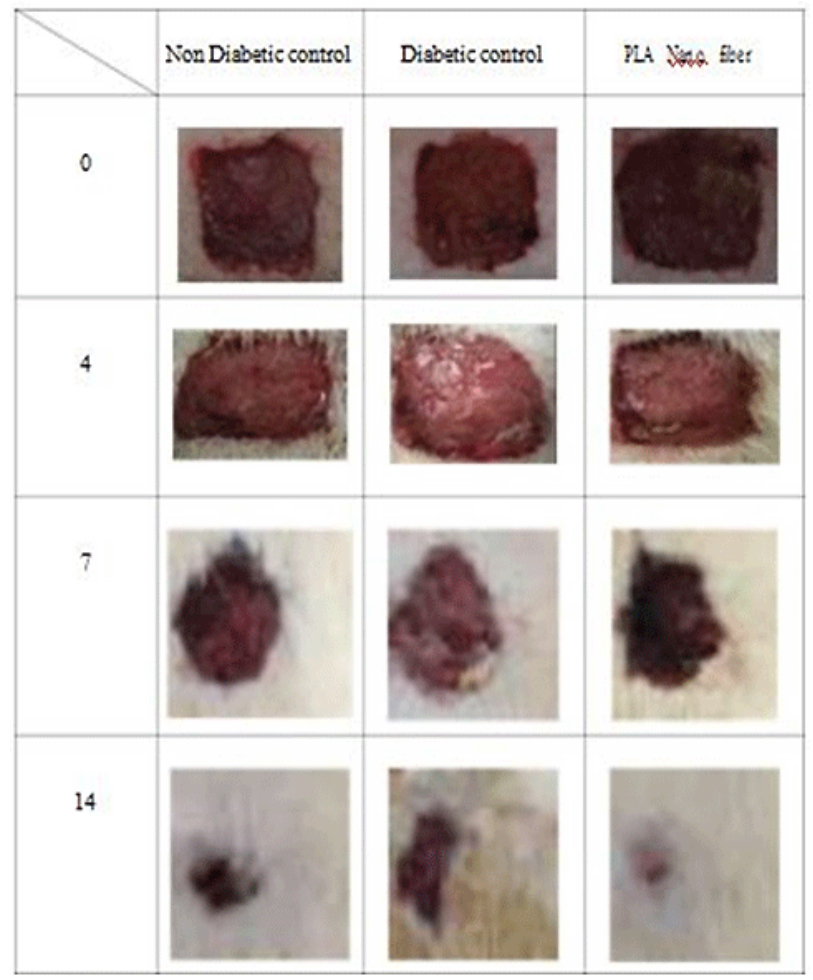

Figure 7: SPhotographs of macroscopic appearances of wound excised from untreated diabetic and non-diabetic rats (control groups) and experimentally diabetic rats treated with PLA Nano Fibers in days 7 and 14 


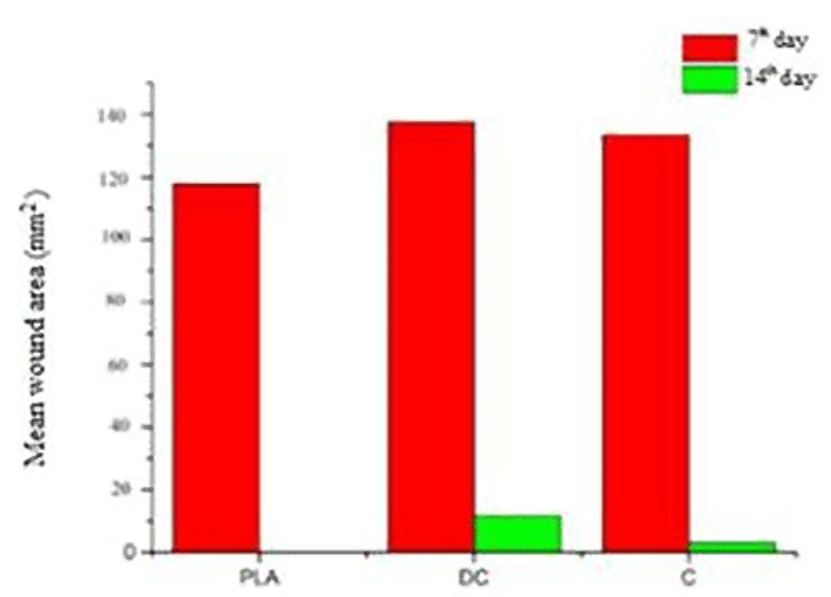

Figure 8: Wound areas in different groups on days 7 and 14C. Non diabetic control: DC, diabetic control: PLA, Poly Latctic and Nano fiber wound dressings

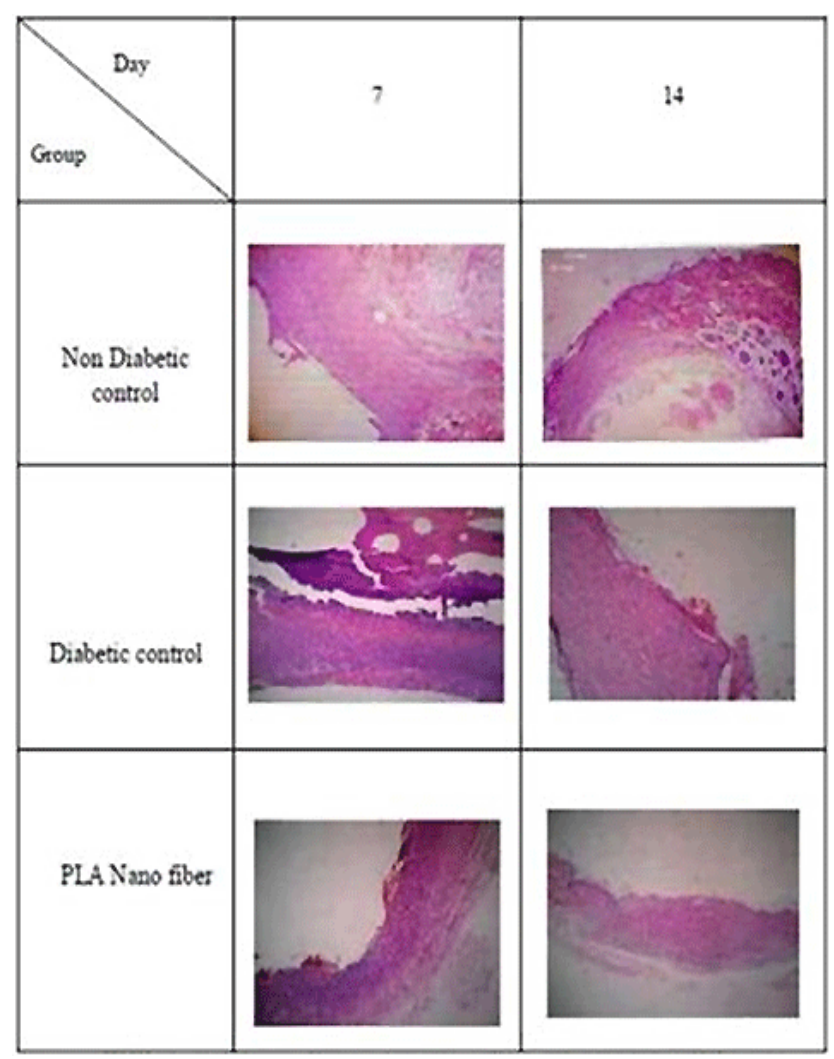

Figure 9: Histological study of wound healing in groups Non diabetic control, Diabetic control and PLA Nano fiber

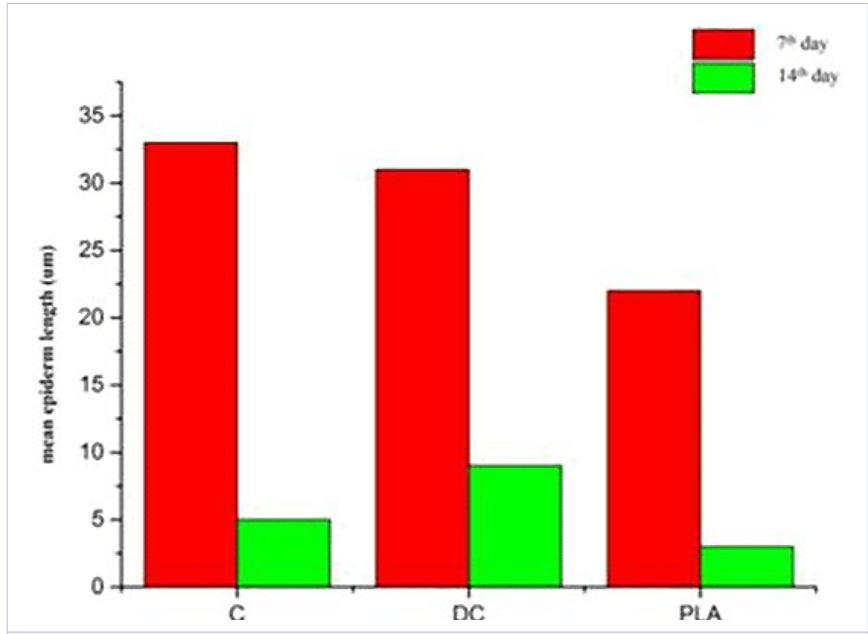

Figure 10: Length of epidermis gap in the study groups at day 7 and day 14

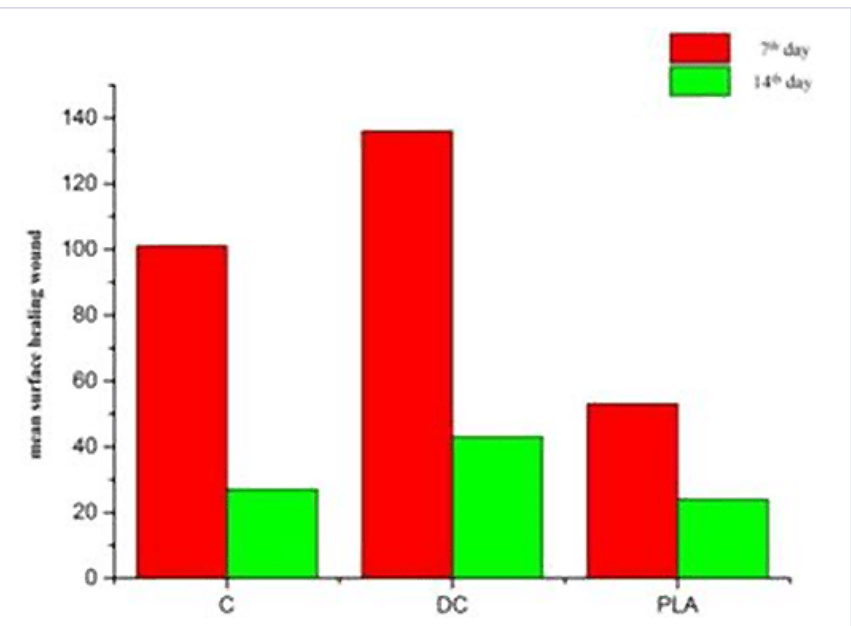

Figure 11: Healing Wound surface of dermis in the study groups at day 7 and 14

\section{Discussion}

Significant technological and scientific advances have been made in the field of electros pinning for the repair and regeneration of tissues including skin. Success in skin tissue engineering is mainly based on regulating cell behavior and tissue progress through the development of a scaffold similar to the natural extracellular matrix that can support cell culture. As a natural extracellular matrix provides an ideal environment chemically, electrically and topographically for cell adhesion and proliferation, tissue engineering also needs a biocompatible scaffold, immunological neutral, driving, biodegradable to support the growth and repair of skin lesions. In general, a suitable 
covering for the wound has features as follows: preventing the infiltration of external infectious agents (some are very small), failure to prevent the entry of oxygen and water, biocompatible, biodegradable, accelerating wound healing, preventing bleeding and failure to create ulcer syndrome. Our studies showed that PLA Nano-fibrous scaffold at a concentration of $2 \%$, due to the small pore size, high biodegradability and wound regenerative properties has a proper feature to be used as a wound dressing.

Today, by imitating the in vivo conditions as much as possible, researchers are trying to improve the efficacy in vitro conditions. Tissue engineering is of great importance to achieve this goal by providing a variety of scaffolds in cell culture environment that can be an imitation of the body's extracellular space. Recent developments in electro spinning method have lead to the production of a continuous solid fiber with diameters in the range of several nanometers by controlling the surface inter-molecular structure. According to the survey, it is found that using electro spinning process is the best method of producing Nano fibers from polymeric materials where the production of Nano fibers is continuously done. Thus, in this study, we used the electro spinning method to prepare PLA Nano fiber scaffold.

Nguyen, et al suggested that Cur-loaded Nano fibers with appropriate Cur concentration are nontoxic and have potential as component of wound-healing patches (13).

Treatment with PLA Glass dressings significantly reduced the wound area, compared with the PLA- or commercial dressingstreated wounds.

The result of the present study revealed that the PLA Nano fibers might be properly used as a wound dressing. The wound healing was accelerated and the Nano fibers sheet was tightly adhered to the wound. In the experiment group PLA Nano fiber, some fragments of the PLA Nano fiber, equal as $1.5 * 1.5$ $\mathrm{cm}^{2}$ were put on the wounds as dressing. The macroscopic and histological findings indicate the improvement of the wound healing process in the $7^{\text {th }}$ and $14^{\text {th }}$ days. The results obtained from the present study indicate a significant reduction in the wound size and improvement of wound healing process of the wounds in artificially diabetic rats after application of PLA Nano fiber scaffold. Therefore, the application of the mentioned compound to help diabetic wound healing can be investigated in further studies particularly in clinical trials. In general, two main reasons might be mentioned for wound healing with Nano fiber PLA: the three-dimensional, network and porous structure of the Nano fiber scaffold which causes the pus and blood moisture absorption and high passage of oxygen over wounds and facilitating wound healing. 2. The chemical structure of Nano fiber PLA and its favorable biological properties such as its biocompatibility and antibacterial nature cause the assimilation of the biochemical environment of the natural tissue and absorption of fibroblasts as well as facilitating wound healing. The instances of wounds are healed after 10 days (14).

\section{References}

1.Shahbazian H, Yazdanpanah L, Latifi SM. Risk assessment of patients with diabetes for foot ulcers according to risk classification consensus of International Working Group on Diabetic Foot (IWGDF). Pak J Med Sci. 2013;29(3):730-734.

2. O’Brien I, Corrall R. Epidemiology of diabetes and its complications. The New England journal of medicine. 1988;318(24):1619-20.

3. Romo T, Al Moutran H, Pearson J, Yalamanchili H, Pafford W, Zoumalan R. Skin wound healing. Medscape Reference. 2012.

4 .Gist S, Tio-Matos I, Falzgraf S, Cameron S, Beebe M. Wound care in the geriatric client. Clin Interv Aging. 2009;4(1):269-287.

5. Ma PX, Langer R. Fabrication of biodegradable polymer foams for cell transplantation and tissue engineering. Tissue engineering methods and protocols. 1999:47-56. doi: 10.1385/0-89603-516-6:47.

6. Shin M ,Ishii O, Sueda T, Vacanti J. Contractile cardiac grafts using a novel nanofibrous mesh. Biomaterials. 2004;25(17):3717-23. doi: 10.1016/j.biomaterials.2003.10.055

7. Vats A, Tolley N, Polak J, Gough J. Scaffolds and biomaterials for tissue engineering: a review of clinical applications. Clinical Otolaryngology. 2003;28(3):165-172.

8. Zhong S, Zhang Y, Lim C. Tissue scaffolds for skin wound healing and dermal reconstruction. Wiley Interdisciplinary Reviews: Nanomedicine and Nanobiotechnology. 2010;2(5):510-525.

9. Almany L, Seliktar D. Biosynthetic hydrogel scaffolds made from fibrinogen and polyethylene glycol for 3D cell cultures. Biomaterials. 2005;26(15):2467-2477. doi: 10.1016/j.biomaterials.2004.06.047

10. Lu X, Wei X, Huang J, Yang L, Zhang G, He G, et al. Supertoughened poly (lactic acid)/polyurethane blend material by in situ reactive interfacial compatibilization via dynamic vulcanization. Industrial \& Engineering Chemistry Research. 2014;53(44):17386-17393.

11. You Y, Lee SW, Youk JH, Min B-M, Lee SJ, Park WH. In vitro degradation behaviour of non-porous ultra-fine poly (glycolic acid)/poly (L-lactic acid) fibres and porous ultra-fine poly (glycolic acid) fibres. Polymer Degradation and Stability. 2005;90(3):441-8. doi: 10.1016/j. polymdegradstab.2005.04.015

12.Jang J-H, Castano O, Kim H-W. Electrospun materials as potential platforms for bone tissue engineering. Advanced drug delivery reviews. 2009;61(12):1065-1083. doi: 10.1016/j.addr.2009.07.008

13. Nguyen TTT, Ghosh C, H wang S-G, Dai Tran L, Park JS. Characteristics of acid) curcumin-loaded poly (lactic nanofibers for wound healing. Journal of materials science. 2013;48(20):7125-7133

14.Gholipour-Kanani A, Bahrami SH, Samadikuchaksaraei A. Novel blend scaffolds from poly (caprolactone) chitosan-poly (vinyl alcohol): Physical, morphological and biological studies. Journal of Biomaterials and Tissue Engineering. 2014;4(3):245-52. doi: $10.1166 /$ jbt.2014.1163 